\title{
Structural Analysis of Self-assembled Nanotubes of Bacteriophage T4 Capsid Protein gp23 by Cryo Electron Microscopy and Mass Spectrometry
}

B. Kükrer Kaletas, ${ }^{1}$ E. Van Duijn, ${ }^{2,3}$ A.J.R. Heck, ${ }^{3}$ R.B.J. Geels, ${ }^{1}$ F. De Haas, ${ }^{4}$ A. van Balen, ${ }^{4}$ S.M. Van der Vies, ${ }^{2}$ A.J. Koster, ${ }^{5}$ L.O. Hertzberger, ${ }^{6}$ R.M.A. Heeren ${ }^{1,3}$

1)Fundamenteel Onderzoek der Materie-Institute for Atomic and Molecular Physics, Kruislaan 407, 1098 SJ Amsterdam, The Netherlands

2)Section Biochemistry and Molecular Biology, Faculty of Science, Vrije Universiteit Amsterdam, De Boelelaan 1083, 1081 HV Amsterdam, The Netherlands

3)Department of Biomolecular Mass Spectrometry, Bijvoet Center for Biomolecular and Utrecht Institute for Pharmaceutical Sciences, Utrecht University, Sorbonnelaan 16, 3584 CA Utrecht, The Netherlands

4) FEI Company, Division of Electron Optics, Eindhoven, The Netherlands

5) Center for Electron Microscopy, Department of Molecular Cell Biology, Leiden University Medical Center, 2300 RA Leiden, The Netherlands

6) University of Amsterdam, Department of Computer Science, Kruislaan 407, 1089 SJ Amsterdam, Netherlands

The capsids of bacterial viruses are self-assembled, nanometer-sized containers, which are striking examples of nature as material engineering. Besides providing chemical protection, capsids are involved in selective packing and injection of the viral genetic material. Bacteriophage T4, a double stranded DNA virus of Escherichia coli, is a widely used object in molecular biology for studying protein folding, oligomeric complex structures and protein assembly mechanisms [1,2]. As revealed by previous studies, the head of T4 predominantly contains the capsid protein gp23 [3]. By investigating polymorphic variants of bacteriophage T4 several characteristic features of the T4 capsid structure have been revealed. One such variant produces the so-called polyhead, which is a long, open-ended nano-sized tube of uncleaved gp23 and is closely related to the prohead structure of T4 making these polyheads ideal for detailed structural analysis [4].

The objective of our project is twofold. The first one is to gain detailed understanding of structural and compositional properties of gp23 polyheads, its components, and assembly intermediates using cryo electron microscopy (cryoEM) and mass spectrometry (MS). Especially, the cryo electron tomography (cryoET) for three-dimensional imaging is a powerful method to reveal the morphology of these nano-sized tubes. The second objective is to investigate the infrastructural requirements of the post-processing and visualization of the large datasets obtained from cryoEM/ET and MS applications. We describe novel ways to integrate the results from two different analytical techniques/methodologies. These studies are performed in the framework of Virtual Laboratory for e-science / Dutch TeleScience Laboratory (VL-e / DUTELLA), which aims to develop a generic and reusable collaborative information management system that allows the proper modeling, management, sharing, and exchange of heterogeneous information generated by diverse e-science domains. It offers scientists remote experiment control, data management facilities and access to distributed resources by providing cross-institutional integration of information and resources in a familiar environment [5]. In this paper, we present preliminary data of novel ways to integrate the results obtained from cryoEM/ET and MS techniques in the VL-e project. 


\section{References}

[1] E. van Duijn, P. J. Bakkes, R.M.A. Heeren, R.H.H. van den Heuvel, H. van Heerikhuizen, S.M. van der Vies, A.J.R. Heck, Nature Methods (2005) 2, 371.

[2] A. Fokine, P. G. Leiman, M. M. Shneider, B. Ahvazi, K. M. Boeshans, A. C. Steven, L. W. Black, V. V. Mesyanzhinov, M. G. Rossmann, PNAS (2005) 102, 7163.

[3] P. G. Leimana, S. Kanamaru, V.V. Mesyanzhinov, F. Arisaka, M. G. Rossmann, CMLS, Cell. Mol. Life Sci. (2003) 60, 2356.

[4] N. H. Olson, M. Gingery, F. A. Eiserling, T. S. Baker, Virology (2001) 279, 385.

[5] H. Afsarmanesh, R.G. Belleman, A. S. Z. Belloum, A. Benabdelkader, J. F. J. van den Brand, G. B. Eijkel, A. Frenkel, C. Garita, D. L. Groep, R. M. A. Heeren, Z. W. Hendrikse, L. O. Hertzberger, J.A. Kaandorp, E. C. Kaletas, V. Korkhov, C.T.A.M. de Laat, P.M.A. Sloot, D. Vasunin, A. Visser, H. H. Yakali, Scientific Programming (2002) 10, 173. 